\title{
Manipulating MiRNA Expression: a Novel Approach for Colon Cancer Prevention and Chemotherapy
}

\author{
Satish Ramalingam • Dharmalingam Subramaniam • \\ Shrikant Anant
}

Published online: 30 January 2015

(C) Springer International Publishing AG 2015

\begin{abstract}
Small non-coding RNA has been implicated in the control of various cellular processes such as proliferation, apoptosis, and differentiation. About $50 \%$ of the miRNA genes are positioned in cancer-associated genomic regions. Several studies have shown that miRNA expression is deregulated in cancer and modulating their expression has reversed the cancer phenotype. Therefore, mechanisms to modulate microRNA (miRNA) activity have provided a novel opportunity for cancer prevention and therapy. In addition, a common cause for development of colorectal cancers is environmental and lifestyle factors. One such factor, diet has been shown to modulate miRNA expression in colorectal cancer patients. In this chapter, we will summarize the work demonstrating that miRNAs are novel promising drug targets for cancer chemoprevention and therapy. Improved delivery, increased stability, and enhanced regulation of off-target effects will overcome the current challenges of this exciting approach in the field of cancer prevention and therapy.
\end{abstract}

Keywords MiRNAs · OncomiRs · Phytochemicals ·

Nanoparticles $\cdot$ Vitamins $\cdot$ Drug delivery

\section{Introduction}

MiRNA, first identified in Caenorhabditis elegans are evolutionarily conserved. They are non-coding RNAs found in most of the eukaryotes, including humans [1,2]. They account

This article is part of the Topical Collection on miRNA and Cancer Prevention and Therapeutic Agents

S. Ramalingam $\cdot$ D. Subramaniam $\cdot$ S. Anant $(\triangle)$

Department of Molecular and Integrative Physiology, and University

of Kansas Cancer Center, University of Kansas School of Medicine,

Kansas City, Kansas, USA

e-mail: sanant@kumc.edu for at least $1-5 \%$ of the human genome and are thought to regulate at least $30 \%$ of the genes [3, 4]. Currently, there were 940 distinct miRNAs identified within the human genome [5, 6]. Although there are several reports demonstrating the important role of the miRNAs in regulation of gene expression, there exists a lacuna about the specific targets and biological functions of the various miRNAs [7, 8]. One thing for certain is that their expression is deregulated in cancer and that $\sim 50 \%$ of genes that encode the miRNAs are located in cancerassociated genomic regions [9] [10]. In addition, modulation of miRNA expression has been shown to reverse the cancer phenotype $[11,12]$.

\section{Genomic Location of MiRNAs and Its Regulation}

Precursors for miRNA are found in various regions of the genome, mostly in the inter-genic regions and also in the introns of the protein coding genes. Until recently, before the identification of these miRNA genes, the intergenic regions within the genome were thought to be junk DNA. MiRNA precursors were rarely also found within exons of the transcripts and in antisense transcripts [13, 14]. Location of the miRNA genes determines the transcriptional units and its regulation. In case of intronic miRNAs that are present within a host gene, in the same orientation will be transcribed with the host transcript by the same promoter $[15,16]$. However, intergenic miRNAs may have their own promoters $[17,18]$.

Transcriptional regulation of miRNA expression is understudied. They are transcribed by both RNA polymerase II and III $[19,20]$. Reports indicate that miRNAs expression is regulated in tissue-specific and developmental-specific manner. In addition, transcription factors and possibly other miRNAs can regulate the transcription of miRNAs in response to both endogenous and exogenous stimuli [21, 22]. Additionally, RNA-binding proteins have been shown to bind 
miRNA precursors and inhibit their maturation and also could direct their degradation [23]. Furthermore, at least $10 \%$ of miRNA expression was regulated through DNA methylation [24]. Recent evidences suggest that miRNAs are regulated in response to hypoxia, hormonal, and dietary changes [25-27].

\section{MiRNA Biogenesis}

Both nuclear and cytoplasmic events are essential for the generation of miRNAs. The first step in the generation of miRNAs is the processing of 5'-capped and 3'-polyadenylated precursor molecules to form primary miRNAs, which are then cleaved by Drosha (RNase III enzyme) and DGCR8 (doublestranded RNA-binding protein) to produce 70-nucleotide hairpin-structured precursor miRNAs [28]. These molecules are then exported from the nucleus to the cytoplasm through the actions of transporter exportin-5 where they are further cleaved by Dicer (endonuclease) and TRBP (RNA-binding protein TAR) to yield miRNA duplex of approximately 2123 nucleotides. In addition, studies suggest that miRNA origin will determine their nuclear pathway [29]. The intergenic miRNA are transcribed by pol II or pol III producing primiRNA, while the coding intronic miRNAs are transcribed by pol II [19, 20, 30].

\section{Gene Silencing Mechanism}

MiRNAs can bind target mRNA to inhibit protein production by one of two distinct mechanisms. The first mechanism is slicer dependent (mRNA cleavage) in which there is endonuclease cleavage of target mRNA by Argonaute 2 (Ago2), which requires extensive base pairing of miRNA with mRNA target [31-33]. The second mechanism of suppressing protein production is slicer independent and involves repressing translation of the mRNA. While this is a reversible process, the former one is irreversible [34-36].

\section{MiRNA and Cancer}

Normal cells undergo genetic changes leading to initiation of malignant transformation followed by progression and metastasis $[37 \cdot, 38]$. Cancer cells proliferate without responding to inhibitory growth signals, evade cell death signals, and induce new blood vessels. Dysregulation of various genes are involved in all the above-mentioned pathways. MiRNAs have been shown to regulate all these cellular pathways, especially those that control cell proliferation, differentiation, and survival. In almost all cancer types, deregulation of miRNA expression has been determined. Until recently, there was a doubt on whether this is a direct cause or an indirect effect of changes in cellular characteristics. However, recent studies have demonstrated that miRNAs can act as pro-oncogenic (oncomirs) or as tumor suppressors [39]. More importantly, miRNAs have been shown to regulate the expression of multiple proteins involved in tumor progression [40]. However, the mechanisms that determine the specificity of targets are currently not well understood.

\section{MiRNAs and Colorectal Cancer}

MiRNAs play a critical role in colorectal cancer progression, invasion, metastasis, and angiogenesis [41]. Colon cancer initiation and progression result from progressive accumulation of genetic modifications in oncogenic and tumor suppressor genes in colonic epithelium. In general initiation of colon cancer has been associated with inactivating mutation in Adenomatus Polyposis Coli and p53 protein, and activation of WNT and K-Ras signaling pathways [42]. Most importantly, more than $60 \%$ of colon cancers have APC inactivation while $50-70 \%$ have p53 mutations. Several miRNAs have been associated with colon cancer progression and metastasis. For example, upregulation of $\mathrm{miR}-135 \mathrm{a} / \mathrm{b}$ results in inhibition of APC mRNA translation [43]. Recent studies have provided insights in to the novel BRAF mutations and its role in colon cancer progression. Although there no reports of miRNAs that target mutated BRAF, a recent study from Nosho and colleagues suggesting the association of miRNA-31 with BRAF mutation may hold promise as a mechanism to inhibit expression of the mutant protein and thereby a novel target for treatment of colorectal cancers [44].

Increased expression of miRNAs expression in colon cancer occurs due to gene amplification, translocation, pleomorphism, or mutation. On the other hand, downregulation has been shown to occur through deletion, promoter methylation, mutations, and aberrant miRNA biogenesis [45]. There were several miRNAs including oncomiRs and tumor suppressor miRNAs that are modulated during the development of colon cancer (Fig. 1). In addition, miRNA expression is regulated in a colon cancer stage-specific manner (Fig. 2). Hence, understanding the order of miRNA regulation during colon cancer progression will enable us to determine treatment strategies. Of the miRNAs that are affected during the colorectal cancers, the key ones are Let-7, miR-21miR-145, and miR17-92 cluster.

\section{Let-7}

This is one of the first miRNAs to be discovered and is highly conserved. Let-7 miRNA can regulate the expression of Ras and c-myc genes, both key factors in colon cancer progression and metastasis. Moreover, Let-7 miRNA regulates p53 and K- 


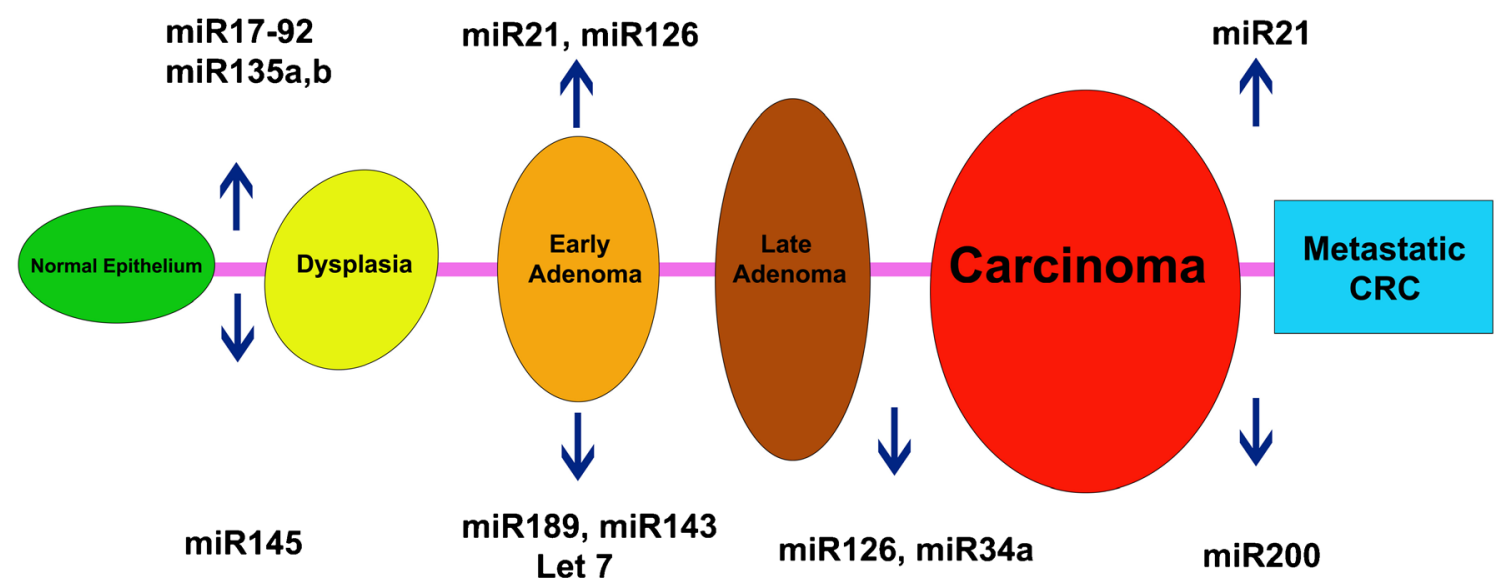

Fig. 1 MicroRNAs during cancer progression. Expression of miRNAs has been observed at various steps during colon cancer progression stages. Specific miRNAs have been identified that regulate initiation,

progression and metastasis. Up arrow indicates the upregulation of the miRNAs, while down arrow refers to the downregulated miRNAs

ras. In fact, the Let-7 miRNA SNP in the K-Ras 3'UTR is prognostic in early-stage colorectal cancer. These results demonstrate that Let-7 functions as tumor suppressor miRNA in colorectal cancer cells [46-48].

\section{MiR-21}

These are oncogenic miRNAs that are found to be overexpressed in colon tumor samples. Initially, it was identified to downregulate the expression of the Phosphatase and Tensin Homolog (PTEN) gene; however, later studies have

Fig. 2 MicroRNAs as tumor promoters and suppressors. The balance between the tumor suppressor miRNAs (green circle) and oncomiRs (red circle) determines the fate of the cell when there are oncogenic signals. However, modulators (phytochemicals, vitamins, minerals, fatty acids, etc.) of these miRNAs are reported to have significant preventive and therapeutic effects in both in vitro and in vivo studies. Further understanding and identifying of these tumor suppressive and oncogenic microRNAs will definitely impart novel preventive and chemotherapeutic agents

found that miR-21 also inhibits other tumor suppressors such as programmed cell death 4 (PDCD4) and tropomyosin 1 (TPM1). Similar to Let-7, miR-21 has been postulated to be a diagnostic and prognostic biomarker in colorectal cancer [49-52].

\section{MiR-145}

The role of miR-145 in colorectal cancer appears to be somewhat controversial. While it was believed to be a tumor suppressor miRNA in colorectal cancer, by targeting both insulin

\section{Modulators}

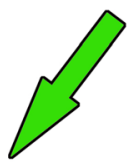

Tumor Suppressors miRs

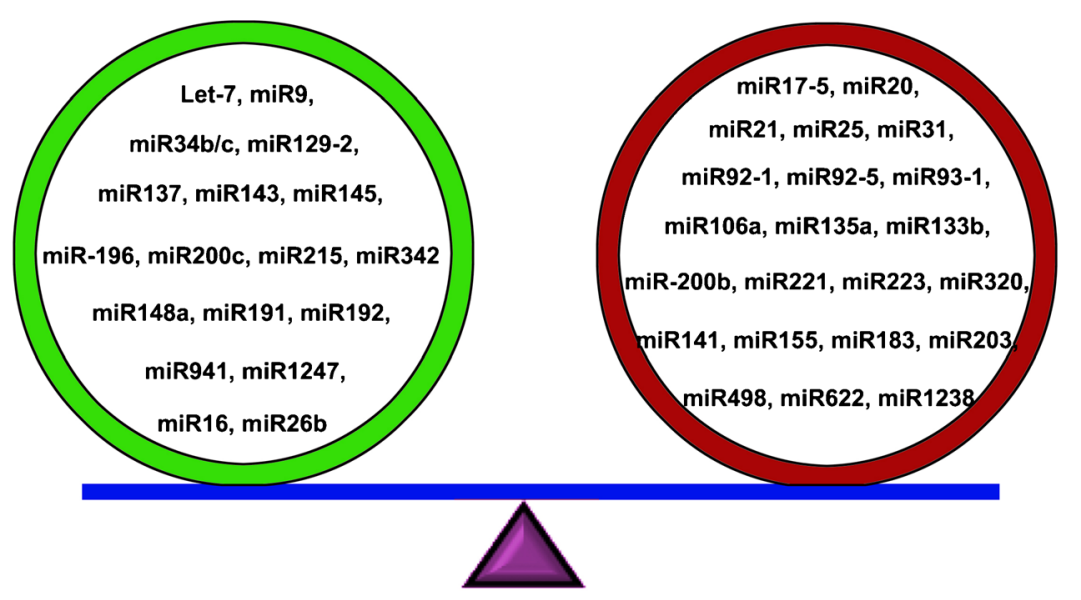


receptor substrate-1 and insulin like growth factor receptor 1 , more recent studies have demonstrated that upregulation of miR-145 could improve the ability of migration and invasion of colorectal cancer cells without affecting proliferation. However, this discrepancy can be explained by more recent studies that suggest that miR145 is highly expressed in mesenchymal cells such as fibroblasts and smooth muscle cells [53, 54].

\section{MiR-17-92}

The miR-17-19 is the highly conserved cluster that produces six mature miRNAs, miR-17, miR-18a, miR-19a, miR-20a, miR-19b-1, and miR-92-1. This cluster has been shown to have both oncogenic and tumor suppressor role. However, in colon cancer it is widely accepted as oncogene as this region is frequently amplified in colon cancers, and that higher levels of miR-17 were associated with poor survival [55]. Furthermore, miR-17 and TNM staging were identified as significant, but independent, prognostic biomarkers in colon cancer [55]. Another study by Tsuchida and colleagues demonstrated that miR-92a was transcribed at higher levels than the other five miRNAs in both adenomas and carcinoma, and that the miRNA targets the anti-apoptotic protein BIM [56]. The miRNAs from this locus appear to have multiple targets. While PTEN was among the first validated target for miR19a and miR-19b-1, BCL2L11 was also shown later on to be targeted by miR-20a, miR-92, miR-19a, and miR-19b-1 [57-59]. Similarly, miR-17 and miR-20a target E2F1 and TGF- $\beta$ receptor II (TGFBRII) [60].

\section{Therapeutic Modulation of MiRNAs for Cancer}

Various pre-clinical studies have reported the possibility of manipulation of oncogenic or tumor-suppressor miRNAs as anticancer therapeutic strategies [61••]. Based on the earlier studies, the miRNA-based therapeutic strategies include inhibition of oncogenic miRNAs or delivery of tumor-suppressor miRNAs.

Several approaches have been reported for inhibiting oncogenic miRNAs including antisense oligonucleotide delivery [62], locked nucleic acid (LNA) constructs delivery [63], miRNA sponge construct delivery, and miRNA masking antisense oligonucleotides technology [64]. In addition, miRNA expression has been suppressed by using inhibitors of oncogenic pathways [65]. On the other hand, for restoration of tumor suppressor miRNAs, approaches used include mature miRNA mimics, miRNA precursors, or pre-miRNA mimics [66-68].

\section{Blocking Oncogenic MiRNAs}

Antisense Oligonucleotides

One of the first methods employed to inhibit expression of oncogenic miRNAs was the use of antisense oligonucleotides. These are competitive inhibitors of the miRNAs, as they bind to the miRNA guide strand and induce their degradation. However, the stability and specificity for target miRNAs along with the binding affinity of antisense oligonucleotides has to be optimized by performing various modifications to the chemical structure of the oligonucleotides [69]. This method of silencing has been shown to be long lasting, specific, and efficient both in vitro and in vivo studies. This strategy was efficient in inhibiting the transformation, metastasis, and drug resistance.

\section{Locked Nucleic Acid (LNA) Constructs}

In an attempt to develop nucleic acid analogs with increased thermal stability and enhanced affinity, LNA nucleotides often referred to as inaccessible RNA were derived. The pentose sugar of ribo- and deoxyribonucleotides are modified by addition of a methylene bridge via a 2'-O atom and the 4'-C atom [70]. Because of this modification, the LNA nucleotides display very high affinity towards RNA and DNA strands, and hence enhance base stacking and backbone pre-organization. Recent reports have demonstrated the use of this technique to successfully knockdown miR-21 in colon cancer cells [71].

\section{Sponge Constructs}

This technique was originally proposed by Philip Sharpe's laboratory at MIT. These are mammalian expression vectors that contain multiple tandem-binding sites of the target miRNA. The basic concept is that the endogenous, targeted miRNA of interest is soaked up by the "sponge" transcript. In addition, to the multiple miRNA binding sites, these "sponge" transcripts encode a bulge at the site normally cleaved by Argonaute 2 thereby facilitating the stable association with ribonucleoprotein complex with the target miRNA [64]. Bu et al. have successfully utilized technique to target miR-34a in colon cancer stem cells $[72,73]$.

\section{Masking Antisense Oligonucleotides}

In this technique, miRNA binding to the target mRNA is prevented by designing antisense oligonucleotides that are fully complimentary to the $3^{\prime}$ untranslated region of the target mRNA. As a result, the miR-mask covers up the miRNA-binding site to hide its target mRNA. To further enhance the capability, a 2'-O-methyl-modification was developed in the oligonucleotides, which also has the added 
effect of significantly reducing off-target effects [62]. The one disadvantage of this approach is that this technology will not be amenable to targeting multiple pathways, something that is desirable in cancer therapy.

Peptide Nucleic Acids (PNAs)

Unlike ribose and deoxyribose sugar backbone, this technology utilizes polyamide backbone with $\mathrm{N}$-(2-aminoethyl)-glycine repeats linked by peptide bonds. This backbone of PNA is neutrally charged and hence binding between the PNA and miRNA is much stronger and resistant to enzyme degradation $[74,75]$. However, to enhance the cytosolic delivery, PNAs need to be coupled with other molecules such as carrier peptides.

\section{Restoration of Tumor-Suppressor MiRNAs}

Re-expressing miRNAs that are downregulated in cancer can be attained either by introducing mature miRNA mimics or plasmids encoding miRNA constructs. Introduction of tumor suppressor miRNAs have been successfully demonstrated in cancer cells to block proliferation, transformation, invasion and migration. In this regard, restoration studies with miR34a and miR-143 have demonstrated decreased cancer progression and metastasis [76, 77]. MiRNA mimics are double stranded RNA molecules having the guide strand similar to the mature miRNA and a passenger strand complimentary to the guide strand. Various modifications have been attempted in the earlier studies to improve its stability, enhance activity and miRNP complex and to increase the protection from degradation.

\section{Delivery Systems and Difficulties}

The two major classifications in the delivery systems are viral and non-viral vectors. Viral systems are further divided into adenoviral and lentiviral vectors. Non-viral vectors encompass lipids (cationic liposomes, neutral lipid emulsion, stable nucleic acid lipid particles) and polymer (polyethylenimine (PEI)), poly (lactic-co-glycolic acid) (PGLA), polyamidoamine (PAMAM)-based nanoparticles.

\section{Viral Vectors}

\section{Adenoviral Vector System}

This is the widely utilized system for in vitro studies to restore tumor suppressor miRNAs, it does not integrate to the genome and are eliminated with very minimal toxicity. Furthermore, tissue-specific targeting is possible because of the availability of multiple serotypes. In addition, adenoviral vector system has high transduction efficiency. However, there are several reports on its utilization in in vivo studies. For example, systemic administration of miR-26a using an adenoviral vector system resulted in cell death and significant reduction in tumor growth. Furthermore, there was no toxicity observed [78]. Also, in another study Let-7 miRNA expressed from an adenoviral vector system, when administered intranasally significantly reduced tumor burden in vivo in the lungs of animals expressing a G12D-activating mutation for the K-ras oncogene [79].

\section{Lentiviral Vectors}

Lentiviral vector systems are potent in delivering miRNAs into a wide range of cell types. These integrate into the host genome and result in long-term expression both in vitro and in vivo. Intratumoral injection of miR-15-16 in a lentiviral vector system has led to the decreased tumor growth [80]. In addition, miR-21 in a lentiviral vector system, when injected intratumorally induced tumor cell death and had better activity in combination with anticancer drugs in inhibiting tumor growth [81].

\section{Non-Viral Vectors}

The non-viral vector system for delivery of miRNA includes lipid-based nanoparticles and polymer-based nanoparticles.

\section{Lipid-Based Nanoparticle}

Liposomes are the most widely used transfection reagent in vitro. However, in in vivo studies, significant success was seen only following significant modifications to the composition and chemical structure of liposomes. Delivery of miRNA34a plasmid using a cationic liposome-mediated system resulted in decreased cancer growth, migration and invasion [82]. Most importantly, there are studies in phase I clinical trial where they are evaluating the efficacy of liposomeformulated miR-34a mimics against liver cancer. Similarly, liposome-formulated delivery of miR-143 and miR-145 when injected systemically resulted in significant inhibitory effect in both subcutaneous and orthotopic mouse models of cancer [83].

Lipoplexes Nucleic acids that are covered with lipids in an organized structure, similar to that seen in a liposome are called lipoplexes. The main reason for doing this is to protect the nucleic acids from degradation. The most common use of lipoplexes is for transferring genetic materials into the cells. With respect to miRNA, pre-miR-107 containing Dime thyldioctadecylammonium (DDAB): cholesterol: $\mathrm{D}-\alpha-$ tocopheryl polyethylene glycol 1000 succinate (TPGS)-based lipoplexes following systemic administration were shown to be effective against head and neck squamous cell cancer [84]. 
Polycationic Liposome-Hyaluronic Acid (LPH)

Chen and colleagues developed a LPH nanoparticle formulation, in which they have included a tumor-targeting singlechain antibody fragment ( $\mathrm{scFv}$ ) for systemic delivery of miRNAs and siRNAs. Using this method, the group was able to successfully deliver siRNAs for Myc, VEGF, and MDM2 along with miR-34a in to murine metastasis model [85].

Dicetyl Phosphate-Tetraethylenepentamine-Based Polycation Liposomes (TEPA-PCL)

This liposome preparation was designed for efficient delivery of siRNA and miRNA. Ando and colleagues employed the miR92a/TEPA-PCL complex to demonstrate the angiogenesis inhibition role of miR92a [86 ${ }^{\bullet}$. Moreover, Asai and colleagues PEGylated the DCP-TEPA-based PCL containing a cholesterolconjugated siRNA and determined its biodistribution in mice bearing a murine colon cancer tumor [87]. There was significant accumulation of the siRNA in the tumor $24 \mathrm{~h}$ after the injection. More importantly, the studies demonstrated the potential feasibility of the approach.

Neutral Lipid Emulsion (NLE) In vivo applications for cationic lipids were limited due to significant toxicity. Therefore, a neutral lipid emulsion was developed. When the emulsion was combined with synthetic miRNA mimics, they form particles in the nanometer diameter with a surface net charge close to zero. Trang and colleagues have successfully demonstrated the utilization of NLE to deliver miR-34a and Let-7 to decrease lung tumor burden [88].

Stable Nucleic Acid Lipid Particles (SNALPs) Stable nucleic acid lipid particles (SNALPs) are microscopic particles approximately $120 \mathrm{~nm}$ in diameter. Here, miRNAs get enclosed by a lipid bilayer comprising a combination of cationic and fusogenic lipids coated with polyethylene glycol (PEG). Costa et al. has demonstrated that chlorotoxin coupled SNALPs were efficient in delivering encapsulated anti-miR-21 oligonucleotides to cultured U87 GBM cells [89]. de Antonellis and colleagues have also used SNALPs to deliver MiR199b-5p a known regulator of Hes-1, a downstream effector of the canonical Notch and noncanonical SHH pathways [90]. Significant downregulation of various target genes were observed demonstrating feasibility of the approach.

In conclusion, lipid-based materials are most widely used and probably the best-studied material for pharmaceutical delivery; therefore, utilizing them for miRNA therapeutic formulation will be advantageous.

There are other alternative materials such as polymerbased nanoparticles that can used to deliver miRNA. These include polyethylenimine (PEI), poly(lactic-co-glycolic acid) (PLGA), and PAMAM dendrimers.
Polyethylenimine

It is the widely studied polymer for gene delivery and it is positively charged because of the protonation of the amine group making it useful for condensing the nucleic acids. Utilizing polyurethane-short branch PEI, miRNA-145 was delivered to treat lung adenocarcinoma and glioblastoma in animal models. PEI systems have been successfully modified for transport of miRNAs across the blood brain barrier [91].

\section{Poly (Lactic-co-Glycolic Acid)}

In contrast with PEI, PLGA-based nanoparticles are highly biocompatible and its physicochemical properties increase its utility in the field for gene delivery [92]. In addition to condensing miRNA and non-specific entry into the cell, PLGA allows the slow release of miRNA over time. PLGA will readily form nanoparticles that can entrap biologically active molecules. It protects miRNAs from degradation and it allows achieving very high loading capacities and provides opportunity for modifications. Babar et al. has demonstrated that anti-miR-155 in PGLA polymer nanoparticle when injected systemically resulted in enhanced therapeutic effect [93].

\section{Polyamidoamine}

Polyamidoamine has positively charged amino group on its surface resulting in enhanced interaction with nucleic acids resulting in a complex that protect the nucleotides from degradation. In addition, its unique construction allows the drugs to fit in the core. Delivery of anti-miR-21 using the polyami doamine has been shown to successfully knockdown miR-21 and resulting in increased sensitivity of glioblastoma cells to taxol treatment [94].

\section{Advantages and Disadvantages}

MiRNA-based therapeutics has been shown to have numerous benefits, such as ability for them to target multiple genes, which play a critical role in colon cancer progression and metastasis. The disadvantages are mainly related to challenges in delivery, stability, possible off-target effects and safety. Another important problem in targeting the miRNAs are there are multiple miRNAs that are known to target single target mRNA and single miRNA has been shown to inhibit multiple mRNAs involved in different pathways. Furthermore, delivering miRNA therapeutics in a tissue-specific manner is still under explored. However, recent studies utilizing various technology to deliver miRNA therapeutics and attempts for chemical modifications of synthetic oligonucleotides has provided more effective means of delivery, has shown great 
therapeutic benefits in multiple in vivo and clinical studies, and has provided enthusiasm to this field [95].

\section{MiRNAs and Diet}

MiRNAs has been shown to regulate various aspects of cancer progression and metastasis that stands as the basis for exploring the ways to modulate miRNA by various chemopreventive and therapeutics agents [96]. Latest evidence from various groups have suggested us that nutrients (vitamins, minerals, fatty acids, etc.) and bioactive food constituents (curcumin, resveratrol, catechins, etc.) protect against cancer through modulation of miRNA expression [97]. In addition, diet has been strongly linked to colon cancer, especially red meat and processed meat, which can increase the risk for colon cancer while fruits and vegetables are shown to significantly decrease the risk [98].

\section{MiRNAs and Essential Nutritional Factors}

Vitamins are vital nutrients that are required to maintain health and have to be taken in sufficient quantity, as our body cannot synthesize them. Several vitamins have been shown to regulate miRNA expression.

\section{Vitamin A}

The multi-functional vitamin A has been shown to regulate vision, growth and development. Vitamin A is an essential dietary factor and has been demonstrated to have inverse correlation with cancer risk [99]; and most importantly in a study from Suphakarn et al. where rats were fed with vitamin Adeficient diet, the rats had enhanced incidence of liver and colon cancer [100]. In addition, trans-retinoic acid, the biologically active form of Vitamin A, was shown to have tumor suppressor activities against various cancer models including lung, bladder, breast, and pancreatic cancer [101]. Interestingly, retinoic acid treatment was shown to modulate the levels of 243 miRNAs in human acute promyelocytic leukemia cells [102]. In addition, another study demonstrated a significant increase in tumor suppressor miRNAs following treatment of retinoic acid in leukemia cells [103].

\section{Vitamin D}

Vitamin D belongs to the group of fat-soluble vitamins and has been shown to regulate intestinal intake of calcium, iron, and zinc. Initially, vitamin D was only associated with bone disease; however, recent studies have recognized its association with cancer. Out of 30 studies on role of vitamin D on colon cancer, a significant number have demonstrated benefit of vitamin D against colon cancer [104, 105]. Treatment of colon cancer cells with calcitriol (active form of vitamin D) led to upregulation of miR-627, which in turn down regulates the expression of the histone demethylase JMJD1A and methylation of histone $\mathrm{H} 3 \mathrm{~K} 9$ and suppressed expression of proliferative factors, such as growth and differentiation factor 15 [106]. In yet another study, vitamin D treatment led to decreased levels of miR-181a and miR-181b, and subsequent increased expression of $\mathrm{p} 27$ and p21, resulting in G1 cell cycle arrest [107]. Furthermore, vitamin D treatment was shown to regulate p53 levels in addition to regulation of miR-182 expression [108].

\section{Vitamin E}

Vitamin E belongs to the lipid-soluble antioxidant vitamins. Vitamin E includes tocopherols and tocotrienols and has demonstrated to protect cell membranes from free radical induced damages. Vitamin $\mathrm{E}$ was shown to decrease the mutation rates, nitrosamine formation, and epithelial cell proliferation. In addition, vitamin $E$ intake in women under the age of 65 has shown strong reduction in colon cancer risk [109]. Furthermore, vitamin $\mathrm{E}$ analogs were shown to possess pro-apoptotic properties and selective killing of malignant cells while being non-toxic to normal cells [110]. Vitamin E was demonstrated to modulate miR-122 and miR-125 expressions and thereby affect the cancer-associated pathways [111]. Additionally, rats fed with vitamin E-deficient diet demonstrated significant decrease in the levels of tumor suppressor miRNAs [111].

\section{Vitamin B}

Vitamin B is the water-soluble vitamin that participates in many coenzyme reactions involved in metabolism. Vitamin $\mathrm{B}$ was shown to activate $\mathrm{p} 53$ and increase $\mathrm{p} 21$ expression in colon cancer cells and mouse colon [112, 113]. In addition, in a rat model of liver cancer, replenishment of folate resulted in increased expression of miR-122 that was associated with inhibition of tumorigenesis [114].

\section{Selenium}

Mineral supplements have gained interest in colon cancer prevention following the studies demonstrating $61 \%$ reduction in colon cancer in a randomized trial with selenium supplementation [115]. In addition, sodium selenite treatment in prostate cancer was shown to increase miR-34b and miR-34c expression [116-118]. In addition, human colorectal adenocarcinoma cells grown in selenium-deficient medium had significantly altered levels of 12 miRNAs, and they have demonstrated that of those 12 miRNAs, miR-185 regulates the expression of GPX2 and SEPSH2 genes [119]. 
Fatty Acids

The role of fatty acids in cancer risk is poorly studied; however, more recently, studies are ongoing to determine the effects of omega-3 polyunsaturated fatty acids in colon cancer. Studies from Davidson et al. have clearly demonstrated that omega-3 PUFAs modulate carcinogen-induced miRNAs in rat colon [120]. In addition, Farago et al. has demonstrated that treatment of glioma cells with PUFAs resulted in increased expression of miRNAs involved in induction of cell death [121].

MiRNAs and Phytochemicals

Fruits and vegetables have abundant phytochemicals that were shown to regulate cancer progression and metastasis. Numerous studies have demonstrated the modulation of miRNAs by these phytochemicals that lead to either abrogation of tumor growth or sensitization of cancer cells to chemotherapeutic agents $[122 \bullet \bullet]$.

We will discuss the list of dietary factors that regulate miRNA expression that can be utilized in the setting of chemoprevention and therapy.

\section{Curcuminoids}

The curcuminoids belong to the class of polyphenols; curcumin is the most extensively studied compound in cancer prevention and therapy. We have earlier published the posttranscriptional regulation of gene expression in cancer by curcumin [123]. There are various reports demonstrating miRNA regulation of curcumin in various types of cancers. Oncogenic miRNA-196 was inhibited by curcumin treatment in gastric cancer [124]; also curcumin treatment was shown to induce PTEN expression by inhibition of miR-200 and miR21 expression [125]. In addition, curcumin treatment has significantly increased tumor suppressor miRNAs in various cancer cells. Even turmeric treatment, has shown to regulate various miRNAs including miR-22, miR-199a*, miR-21, miR200, miR-16, and they have shown to target various cancer related genes such as SP1, ESR1, and PTEN [124].

\section{Resveratrol}

Resveratrol, a chemopreventive phytochemical from grapes, was shown to be effectively inhibiting colon cancer cells growth [126]. There are several reports suggesting that resveratrol modulate miRNAs including miR-21, miR-196a, miR25, miR-17, and miR-92a-2 [127]. In addition, resveratrol was shown to regulate Dicer, PDCD4, and PTEN through miR663 regulation in colon cancer cells; moreover, resveratrol downregulated oncogenic miR-155 in a miR-663-dependent manner [128]. Furthermore, resveratrol modulated the levels of miRNAs targeting tumor-suppressor proteins in SW480 colon cancer cell lines [129]. A recent report suggested that resveratrol exhibits anti-colon cancer activity through inhibition of oncogenic miR-27a [130]. Kumazaki et al. demonstrated that resveratrol exerted its anticancer effect through regulation of miR-34a/E2F3/Sirt1 cascade [131].

\section{Catechins}

Epigallocatechin-3-gallate (EGCG) is the major polyphenol present in green tea; however, there are other catechins such as epicatechin, epicatechin gallate, and apigallocatechin that have similar features [132]. Several preclinical studies have demonstrated EGCG's anti-colon cancer activity [133]. Baselga-Escudero and colleagues have demonstrated that EGCG directly binds to miR-33a and miR-122, which is a novel posttranscriptional mechanism for the polyphenols [134]. Furthermore, EGCG in combination with N-(4hydroxyphenyl) retinamide has significantly inhibited the expression of oncogenic miRNAs such as miR-92, miR-93, and miR-106b, while increasing the expression of tumor suppressor miRNAs like miR-7-1, miR-34a, and miR-99a [135]. In addition, EGCG treatment resulted in increased expression of tumor suppressor miR-16 that is known to induce apoptosis through targeting Bcl2 expression [136].

\section{Genistein}

Genistein is a phytoestrogenic isoflavonoid that was shown to have potent anticancer activity by affecting cell proliferation and inducing apoptosis [137]. It has been shown to upregulate tumor-suppressor miR-574-3p in prostate cancer cells and miR-574-3p target genes include RAC1, EGFR, and EP300 [138]. In addition, $\mathrm{Xu}$ et al. has demonstrated that genistein targets oncogenic miR-27a in ovarian cancer cells [139]. In renal cancer cell lines, genistein was shown to upregulate the expression of tumor suppressor miRNA miR-23b [140]

\section{Isothiocyanates}

Isothiocyanates (ITCs) present in cruciferous vegetables were shown to possess potent anticancer activity using various in vitro and in vivo studies [141]. Sulforaphane is an ITC obtained from broccoli and has shown to inhibit epithelial and mesenchymal transition through regulation of ZEB1 and Snail through increased expression of miR-200c [142]. Another agent, phenethyl isothiocyanate (PEITC), has been shown to regulate the expression of miRNAs that were altered due to cigarette smoke exposure. In the study, they have identified that PEITC regulate various miRNA expression, including miR-192, let-7a, let-7c, miR-146, miR-123, miR-222, and miR-99b [143]. 
Indole-3-carbinol (I3C) and 3,3'-Diindolylmethane (DIM)

I3C is a glucosinolates found in the brassica family of vegetables such as broccoli, cauliflower, and brussel sprouts and DIM is a product obtained during condensation reaction of I3C in the gut. Both I3C and DIM have been reported to possess potent anticancer activity through its ability to regulate cancer cell cycle, induction of apoptosis and major oncogenic signaling pathways [144]. In colon and pancreatic cancer cell lines, DIM treatment leads to induction in the expression of miR-146a thereby targeting EGFR, IRAK1, and NFkB mRNAs [145]. In a mouse model of lung cancer, I3C treatment resulted in reducing the carcinogen-induced expression of miRNAs (miR-21, miR31, miR-130a, miR-146b, and miR377) [146]. In another study, I3C-induced sensitivity of cancer cells to gemcitabine was found to be suppressed when miR-21 was ectopically overexpressed, suggesting that downregulation of miR 21 by $\mathrm{I} 3 \mathrm{C}$ is essential for its action on induced chemosensitivity [147]. Finally, reports also revealed that DIM treatment resulted in downregulation of miR-221 and thereby increasing the expression of its target gene expression such as PTEN, p27, and PUMA [148].

\section{Ellagitannin}

Ellagitannins, a polyphenolic compound isolated from strawberries and raspberries was reported to have significant anticancer activity [149]. Liver cancer cell lines when treated with ellagitannin increased the expression of 17 miRNAs while inhibiting 8 suggesting that these microRNAs are at least partly responsible for the antiproliferative and multigene regulatory mechanism of action induced by the compound on HepG2 cancer cells [150].

Altogether, these studies support the potential for these natural phytochemicals along with vitamins, fatty acids, and trace elements in modulating the miRNA expression that could be beneficial in the colon cancer prevention and therapy development.

\section{Conclusions and Future Perspectives}

The role of microRNAs affecting cancer is now well documented. However, its utility as a therapeutic or preventive agent is yet to be fully realized. Given that miRNAs have been shown to regulate various stages of cancer development and the vast amount of information about the multiple miRNAs and their targets have already been accumulated, the time is right to develop novel chemopreventive and therapeutic modalities. This will include targeted delivery and reducing adverse effects due to toxicity. There is significant promise for use of viral and non-viral agents for delivering the miRNA, but then again getting them to honing in to the sites of the tumor is going to be important. Furthermore, moderating miRNA levels using mimics is another exciting area, but non-specific targets need to be reduced. There is also a need to improve the stability of the plasmids and minimize the induction of immune response to advance the use of miRNA in colon cancer prevention and therapeutics. Finally, several dietary agents have been shown to be efficient modulators of miRNA profiles in cancer. However, there are concerns such as in vivo bioavailability of the compounds, specificity in targeting, and the under studied metabolites. Once these issues are overcome, the promise of natural compounds modulating miRNA expression and thereby suppressing various cancer promoting pathways is a possibility.

Acknowledgments The study was supported by grants from the National Institutes of Health and the Kansas Bioscience Authority.

\section{Compliance with Ethical Guidelines}

Conflict of Interest Satish Ramalingam, Dharmalingam Subramaniam and Shrikant Anant have no conflict of interest.

Human and Animal Rights and Informed Consent This article does not contain any studies with human or animal subjects performed by any of the authors.

\section{References}

Papers of particular interest, published recently, have been highlighted as:

- Of importance

•- Of major importance

1. Zhou X, Yang PC. MicroRNA: a small molecule with a big biological impact. Micro RNA. 2012;1(1):1.

2. Perron MP, Provost P. Protein interactions and complexes in human microRNA biogenesis and function. Front Biosci : J Virtual Libr. 2008;13:2537-47.

3. Macfarlane LA, Murphy PR. MicroRNA: biogenesis, function and role in cancer. Curr Genomics. 2010;11(7):537-61.

4. Rajewsky N. L (ou)sy miRNA targets? Nat Struct Mol Biol. 2006;13(9):754-5.

5. Griffiths-Jones S. The microRNA registry. Nucleic Acids Res. 2004;32(Database issue):D109-11.

6. Cullen BR. Transcription and processing of human microRNA precursors. Mol Cell. 2004;16(6):861-5.

7. Ambros V. The functions of animal microRNAs. Nature. 2004;431(7006):350-5.

8. Krutzfeldt $J$ et al. Silencing of microRNAs in vivo with 'antagomirs'. Nature. 2005;438(7068):685-9.

9. Huppi $\mathrm{K}$ et al. The identification of microRNAs in a genomically unstable region of human chromosome 8q24. Mol Cancer Res : MCR. 2008;6(2):212-21.

10. Pomerantz MM et al. Evaluation of the $8 \mathrm{q} 24$ prostate cancer risk locus and MYC expression. Cancer Res. 2009;69(13):5568-74.

11. Wu W. Modulation of microRNAs for potential cancer therapeutics. Methods Mol Biol. 2011;676:59-70. 
12. Matsushima K et al. MiRNA-205 modulates cellular invasion and migration via regulating zinc finger E-box binding homeobox 2 expression in esophageal squamous cell carcinoma cells. J Transl Med. 2011;9:30.

13. Gibbs WW. The unseen genome: gems among the junk. Sci Am. 2003;289(5):26-33.

14. Fiorucci $\mathrm{G}$ et al. Cancer regulator microRNA: potential relevance in diagnosis, prognosis and treatment of cancer. Curr Med Chem. 2012;19(4):461-74.

15. Rodriguez A, Griffiths-Jones S, Ashurst JL, Bradley A. Identification of mammalian microRNA host genes and transcription units. Genome Res. 2004;14(10A):1902-10.

16. Ying SY, Chang CP, Lin SL. Intron-mediated RNA interference, intronic microRNAs, and applications. Methods Mol Biol. 2010;629:205-37.

17. Lagos-Quintana M, Rauhut R, Meyer J, Borkhardt A, Tuschl T. New microRNAs from mouse and human. RNA. 2003;9(2): 175-9.

18. Saini HK, Griffiths-Jones S, Enright AJ. Genomic analysis of human microRNA transcripts. Proc Natl Acad Sci U S A. 2007;104(45):17719-24.

19. Lee Y et al. MicroRNA genes are transcribed by RNA polymerase II. EMBO J. 2004;23(20):4051-60.

20. Borchert GM, Lanier W, Davidson BL. RNA polymerase III transcribes human microRNAs. Nat Struct Mol Biol. 2006;13(12): 1097-101.

21. Pillai RS, Bhattacharyya SN, Filipowicz W. Repression of protein synthesis by miRNAs: how many mechanisms? Trends Cell Biol. 2007;17(3):118-26.

22. Bartel DP. MicroRNAs: genomics, biogenesis, mechanism, and function. Cell. 2004;116(2):281-97.

23. Viswanathan SR, Daley GQ. Lin28: A microRNA regulator with a macro role. Cell. 2010;140(4):445-9.

24. Han L, Witmer PD, Casey E, Valle D, Sukumar S. DNA methylation regulates MicroRNA expression. Cancer Biol Therapy. 2007;6(8):1284-8.

25. Kulshreshtha R et al. A microRNA signature of hypoxia. Mol Cell Biol. 2007;27(5):1859-67.

26. Fiedler SD, Carletti MZ, Hong X, Christenson LK. Hormonal regulation of MicroRNA expression in periovulatory mouse mural granulosa cells. Biol Reprod. 2008;79(6):1030-7.

27. Davis CD, Ross SA. Evidence for dietary regulation of microRNA expression in cancer cells. Nutr Rev. 2008;66(8):477-82.

28. Han $\mathrm{J}$ et al. Posttranscriptional crossregulation between Drosha and DGCR8. Cell. 2009;136(1):75-84.

29. Chendrimada TP et al. TRBP recruits the Dicer complex to Ago2 for microRNA processing and gene silencing. Nature. 2005;436(7051): $740-4$.

30. Han $\mathrm{J}$ et al. Molecular basis for the recognition of primary microRNAs by the Drosha-DGCR8 complex. Cell. 2006;125(5): 887-901.

31. Meister $\mathrm{G}$ et al. Human Argonaute2 mediates RNA cleavage targeted by miRNAs and siRNAs. Mol Cell. 2004;15(2):185-97.

32. Liu $\mathrm{J}$ et al. Argonaute 2 is the catalytic engine of mammalian RNAi. Science. 2004;305(5689):1437-41.

33. Valencia-Sanchez MA, Liu J, Hannon GJ, Parker R. Control of translation and mRNA degradation by miRNAs and siRNAs. Genes Dev. 2006;20(5):515-24.

34. Brengues M, Teixeira D, Parker R. Movement of eukaryotic mRNAs between polysomes and cytoplasmic processing bodies. Science. 2005;310(5747):486-9.

35. Maroney PA, Yu Y, Fisher J, Nilsen TW. Evidence that microRNAs are associated with translating messenger RNAs in human cells. Nat Struct Mol Biol. 2006;13(12):1102-7.

36. Maroney PA, Yu Y, Nilsen TW. MicroRNAs, mRNAs, and translation. Cold Spring Harb Symp Quant Biol. 2006;71:531-5.
37. Binefa G, Rodriguez-Moranta F, Teule A, Medina-Hayas M. Colorectal cancer: from prevention to personalized medicine. World J Gastroenterol : WJG. 2014;20(22):6786-808. In this review, the author's have highlighted key findings in the field of colorectal cancer and discussed various aspects from prevention to personalized medicine. In addition, they have provided information on recent advances in molecular biology and the genetic classifications of colorectal cancer. Furthermore, they have provided insights on ways to improve the colon cancer treatment in the coming years.

38. Bouyssou JM et al. Regulation of microRNAs in cancer metastasis. Biochim Biophys Acta. 2014;1845(2):255-65.

39. Huang $J$ et al. MicroRNAs as oncogenes or tumour suppressors in oesophageal cancer: potential biomarkers and therapeutic targets. Cell Prolif. 2014;47(4):277-86.

40. Kasinski AL, et al. (2014) A combinatorial microRNA therapeutics approach to suppressing non-small cell lung cancer. Oncogene.

41. Tokarz P, Blasiak J. The role of microRNA in metastatic colorectal cancer and its significance in cancer prognosis and treatment. Acta Biochim Pol. 2012;59(4):467-74.

42. Aslam MI, Patel M, Singh B, Jameson JS, Pringle JH. MicroRNA manipulation in colorectal cancer cells: from laboratory to clinical application. J Transl Med. 2012;10:128.

43. Slaby O, Svoboda M, Michalek J, Vyzula R. MicroRNAs in colorectal cancer: translation of molecular biology into clinical application. Mol Cancer. 2009;8:102.

44. Nosho K et al. Association of microRNA-31 with BRAF mutation, colorectal cancer survival and serrated pathway. Carcinogenesis. 2014;35(4):776-83.

45. Esquela-Kerscher A, Slack FJ. Oncomirs-microRNAs with a role in cancer. Nature reviews. Cancer. 2006;6(4):259-69.

46. Sha $\mathrm{D}$ et al. Association study of the let-7 miRNA-complementary site variant in the $3^{\prime}$ untranslated region of the KRAS gene in stage III colon cancer (NCCTG N0147 Clinical Trial). Clin Cancer Res : Off J Am Assoc Cancer Res. 2014;20(12):3319-27.

47. King CE et al. LIN28B fosters colon cancer migration, invasion and transformation through let-7-dependent and -independent mechanisms. Oncogene. 2011;30(40):4185-93.

48. Akao Y, Nakagawa Y, Naoe T. let-7 microRNA functions as a potential growth suppressor in human colon cancer cells. Biol Pharm Bull. 2006;29(5):903-6.

49. Deng J et al. Targeting miR-21 enhances the sensitivity of human colon cancer HT-29 cells to chemoradiotherapy in vitro. Biochem Biophys Res Comm. 2014;443(3):789-95.

50. Oue $\mathrm{N}$ et al. High miR-21 expression from FFPE tissues is associated with poor survival and response to adjuvant chemotherapy in colon cancer. Int J Cancer J Int Cancer. 2014;134(8):1926-34.

51. Roy S, Yu Y, Padhye SB, Sarkar FH, Majumdar AP. Difluorinated-curcumin (CDF) restores PTEN expression in colon cancer cells by down-regulating miR-21. PLoS One. 2013;8(7):e68543.

52. Faltejskova $\mathrm{P}$ et al. Clinical correlations of miR-21 expression in colorectal cancer patients and effects of its inhibition on DLD1 colon cancer cells. Int J Color Dis. 2012;27(11):1401-8.

53. Zhang J et al. Putative tumor suppressor miR-145 inhibits colon cancer cell growth by targeting oncogene Friend leukemia virus integration 1 gene. Cancer. 2011;117(1):86-95.

54. Zhu $\mathrm{H}$ et al. EGFR signals downregulate tumor suppressors miR143 and miR-145 in Western diet-promoted murine colon cancer: role of G1 regulators. Mol Cancer Res : MCR. 2011;9(7):960-75.

55. Yu G et al. Prognostic values of the miR-17-92 cluster and its paralogs in colon cancer. J Surg Oncol. 2012;106(3):232-7.

56. Tsuchida A et al. miR-92 is a key oncogenic component of the miR-17-92 cluster in colon cancer. Cancer Sci. 2011;102(12): 2264-71. 
57. Peric D, Chvalova K, Rousselet G. Identification of microprocessordependent cancer cells allows screening for growth-sustaining micro-RNAs. Oncogene. 2012;31(16):2039-48.

58. Liang Z, Li Y, Huang K, Wagar N, Shim H. Regulation of miR-19 to breast cancer chemoresistance through targeting PTEN. Pharm Res. 2011;28(12):3091-100.

59. Olive Vet al. miR-19 is a key oncogenic component of mir-17-92. Genes Dev. 2009;23(24):2839-49.

60. Tili E et al. GAM/ZFp/ZNF512B is central to a gene sensor circuitry involving cell-cycle regulators, TGF \{beta\} effectors, Drosha and microRNAs with opposite oncogenic potentials. Nucleic Acids Res. 2010;38(21):7673-88.

$61 . \bullet$ Humphreys KJ et al. Dietary manipulation of oncogenic microRNA expression in human rectal mucosa: a randomized trial. Cancer Prev Res. 2014;7(8):786-95. Intake of high red meat is associated with increased colorectal cancer risk. In addition, elevated oncogenic microRNAs have been shown to induce tumorigenesis. In this article, for the first time, it was demonstrated that dietary manipulation of microRNA by butyrylated resistant starch supplementation reduced the risk of human rectal cancer associated with high red meat intake in a randomized trial.

62. Xiao $\mathrm{J}$ et al. Novel approaches for gene-specific interference via manipulating actions of microRNAs: examination on the pacemaker channel genes HCN2 and HCN4. J Cell Physiol. 2007;212(2):285-92.

63. Veedu RN, Wengel J. Locked nucleic acids: promising nucleic acid analogs for therapeutic applications. Chem Biodivers. 2010;7(3):536-42.

64. Ebert MS, Neilson JR, Sharp PA. MicroRNA sponges: competitive inhibitors of small RNAs in mammalian cells. Nat Methods. 2007;4(9):721-6.

65. Gumireddy K et al. Small-molecule inhibitors of microrna miR-21 function. Angew Chem. 2008;47(39):7482-4.

66. Hermeking H. The miR-34 family in cancer and apoptosis. Cell Death Differ. 2010;17(2):193-9.

67. Cole KA et al. A functional screen identifies miR-34a as a candidate neuroblastoma tumor suppressor gene. Mol Cancer Res : MCR. 2008;6(5):735-42.

68. Ng EK et al. MicroRNA-143 targets DNA methyltransferases 3A in colorectal cancer. Br J Cancer. 2009;101(4):699-706.

69. Choi WY, Giraldez AJ, Schier AF. Target protectors reveal dampening and balancing of Nodal agonist and antagonist by miR-430 . Science. 2007;318(5848):271-4.

70. Kauppinen S, Vester B, Wengel J. Locked nucleic acid: highaffinity targeting of complementary RNA for RNomics. Handb Exp Pharmacol. 2006;173:405-22.

71. Valeri $\mathrm{N}$ et al. MicroRNA-21 induces resistance to 5-fluorouracil by down-regulating human DNA MutS homolog 2 (hMSH2). Proc Natl Acad Sci U S A. 2010;107(49):21098-103.

72. Dias BG et al. Amygdala-Dependent Fear Memory Consolidation via miR-34a and Notch Signaling. Neuron. 2014;83(4):906-18.

73. $\mathrm{Bu} \mathrm{P}$ et al. A microRNA miR-34a-regulated bimodal switch targets Notch in colon cancer stem cells. Cell Stem Cell. 2013;12(5): 602-15.

74. Veldhoen S, Laufer SD, Restle T. Recent developments in peptidebased nucleic acid delivery. Int J Mol Sci. 2008;9(7):1276-320.

75. Fabbri $\mathrm{E}$ et al. Modulation of the biological activity of microRNA210 with peptide nucleic acids (PNAs). Chem Med Chem. 2011;6(12):2192-202.

76. Mudduluru $\mathrm{G}$ et al. Regulation of Axl receptor tyrosine kinase expression by miR-34a and $\mathrm{miR}-199 \mathrm{a} / \mathrm{b}$ in solid cancer. Oncogene. 2011;30(25):2888-99.

77. Nakagawa Y, Iinuma M, Naoe T, Nozawa Y, Akao Y. Characterized mechanism of alpha-mangostin-induced cell death: caspase-independent apoptosis with release of endonuclease-G from mitochondria and increased miR-143 expression in human colorectal cancer DLD-1 cells. Bioorg Med Chem. 2007;15(16): 5620-8.

78. Kota $\mathrm{J}$ et al. Therapeutic microRNA delivery suppresses tumorigenesis in a murine liver cancer model. Cell. 2009;137(6): 1005-17.

79. Trang $\mathrm{P}$ et al. Regression of murine lung tumors by the let-7 microRNA. Oncogene. 2010;29(11):1580-7.

80. Bonci $\mathrm{D}$ et al. The miR-15a-miR-16-1 cluster controls prostate cancer by targeting multiple oncogenic activities. Nat Med. 2008;14(11):1271-7.

81. Sicard F, Gayral M, Lulka H, Buscail L, Cordelier P. Targeting miR-21 for the therapy of pancreatic cancer. Mol Therapy : J Am Soc Genet Therapy. 2013;21(5):986-94.

82. $\mathrm{Li} \mathrm{L}$ et al. Targeted expression of miR-34a using the T-VISA system suppresses breast cancer cell growth and invasion. Mol Therapy : J Am Soc Genet Therapy. 2012;20(12):2326-34.

83. Pramanik D et al. Restitution of tumor suppressor microRNAs using a systemic nanovector inhibits pancreatic cancer growth in mice. Mol Cancer Ther. 2011;10(8):1470-80.

84. Piao L et al. Lipid-based nanoparticle delivery of Pre-miR-107 inhibits the tumorigenicity of head and neck squamous cell carcinoma. Mol Therapy : J Am Soc Genet Therapy. 2012;20(6): 1261-9.

85. Chen Y, Zhu X, Zhang X, Liu B, Huang L. Nanoparticles modified with tumor-targeting scFv deliver siRNA and miRNA for cancer therapy. Mol Therapy : J Am Soc Genet Therapy. 2010;18(9):1650-6.

86. Ando $\mathrm{H}$ et al. Development of a miR-92a delivery system for antiangiogenesis-based cancer therapy. J Genet Med. 2013;15(1):20-7. MicroRNAs appears to be the promising targets for treatment of cancer. Furthermore, various studies have demonstrated that miRNAs mediate angiogenesis and metastasis by regulating the expression of target genes. In this study, they have developed a miRNA delivery system and demonstrated its ability to inhibit angiogenesis and to suppress tumor growth.

87. Asai T et al. Dicetyl phosphate-tetraethylenepentamine-based liposomes for systemic siRNA delivery. Bioconjug Chem. 2011;22(3):429-35.

88. Trang $\mathrm{P}$ et al. Systemic delivery of tumor suppressor microRNA mimics using a neutral lipid emulsion inhibits lung tumors in mice. Mol Therapy : J Am Soc Genet Therapy. 2011;19(6):1116-22.

89. Costa PM et al. Tumor-targeted Chlorotoxin-coupled Nanoparticles for Nucleic Acid Delivery to Glioblastoma Cells: A Promising System for Glioblastoma Treatment. Mol Therapy Nucleic Acids. 2013;2:e100.

90. de Antonellis P et al. MicroRNA 199b-5p delivery through stable nucleic acid lipid particles (SNALPs) in tumorigenic cell lines. Naunyn Schmiedeberg's Arch Pharmacol. 2013;386(4):287-302.

91. Yang YP et al. Inhibition of cancer stem cell-like properties and reduced chemoradioresistance of glioblastoma using microRNA145 with cationic polyurethane-short branch PEI. Biomaterials. 2012;33(5):1462-76.

92. Bala I, Hariharan S, Kumar MN. PLGA nanoparticles in drug delivery: the state of the art. Crit Rev Ther Drug Carrier Syst. 2004;21(5):387-422.

93. Babar IA et al. Nanoparticle-based therapy in an in vivo microRNA-155 (miR-155)-dependent mouse model of lymphoma. Proc Natl Acad Sci U S A. 2012;109(26):E1695-704.

94. Ren $\mathrm{Y}$ et al. Co-delivery of as-miR-21 and 5-FU by poly(amidoamine) dendrimer attenuates human glioma cell growth in vitro. J Biomater Sci Polym Ed. 2010;21(3):303-14.

95. Costa PM, Pedroso de Lima MC. MicroRNAs as Molecular Targets for Cancer Therapy: On the Modulation of MicroRNA Expression. Pharmaceuticals. 2013;6(10):1195-220.

96. Parasramka MA, Ho E, Williams DE, Dashwood RH. MicroRNAs, diet, and cancer: new mechanistic insights on the 
epigenetic actions of phytochemicals. Mol Carcinog. 2012;51(3): 213-30.

97. Sarkar FH, Li Y, Wang Z, Kong D, Ali S. Implication of microRNAs in drug resistance for designing novel cancer therapy. Drug Resist Updat : Rev Commentaries Antimicrob Anticancer Chemother. 2010;13(3):57-66.

98. Milenkovic D, Jude B, Morand C. miRNA as molecular target of polyphenols underlying their biological effects. Free Radic Biol Med. 2013;64:40-51.

99. Wu Y, et al. (2014) Association between vitamin A, retinol intake and blood retinol level and gastric cancer risk: A meta-analysis. Clinical nutrition.

100. Suphakarn VS, Newberne PM, Goldman M. Vitamin A and aflatoxin: effect on liver and colon cancer. Nutr Cancer. 1983;5(1):41-50.

101. Sun SY, Lotan R. Retinoids and their receptors in cancer development and chemoprevention. Crit Rev Oncol Hematol. 2002;41(1): $41-55$.

102. Rossi A et al. Non-coding RNAs change their expression profile after Retinoid induced differentiation of the promyelocytic cell line NB4. BMC Res Notes. 2010;3:24.

103. Garzon $\mathrm{R}$ et al. MicroRNA gene expression during retinoic acidinduced differentiation of human acute promyelocytic leukemia. Oncogene. 2007;26(28):4148-57.

104. Garland CF et al. The role of vitamin D in cancer prevention. Am J Public Health. 2006;96(2):252-61.

105. Garland CF, Garland FC. Do sunlight and vitamin D reduce the likelihood of colon cancer? Int J Epidemiol. 2006;35(2):217-20.

106. Padi SK, Zhang Q, Rustum YM, Morrison C, Guo B. MicroRNA627 mediates the epigenetic mechanisms of vitamin D to suppress proliferation of human colorectal cancer cells and growth of xenograft tumors in mice. Gastroenterology. 2013;145(2):437-46.

107. Wang X, Gocek E, Liu CG, Studzinski GP. MicroRNAs181 regulate the expression of p27Kip1 in human myeloid leukemia cells induced to differentiate by 1,25-dihydroxyvitamin D3. Cell Cycle. 2009;8(5):736-41.

108. Peng $X$ et al. Protection against cellular stress by 25 hydroxyvitamin D3 in breast epithelial cells. J Cell Biochem. 2010;110(6):1324-33.

109. Bostick RM et al. Reduced risk of colon cancer with high intake of vitamin E: the Iowa Women's Health Study. Cancer Res. 1993;53(18):4230-7.

110. Wang XF, Witting PK, Salvatore BA, Neuzil J. Vitamin E analogs trigger apoptosis in HER2/erbB2-overexpressing breast cancer cells by signaling via the mitochondrial pathway. Biochem Biophys Res Commun. 2005;326(2):282-9.

111. Gaedicke $\mathrm{S}$ et al. Vitamin $\mathrm{E}$ dependent microRNA regulation in rat liver. FEBS Lett. 2008;582(23-24):3542-6.

112. Zhang P, Suidasari S, Hasegawa T, Yanaka N, Kato N. Vitamin $\mathrm{B}(6)$ activates $\mathrm{p} 53$ and elevates $\mathrm{p} 21$ gene expression in cancer cells and the mouse colon. Oncol Rep. 2014;31(5):2371-6.

113. Zhong H, et al. (2014) Association of vitamin D receptor gene polymorphism with the risk of lung cancer: a meta-analysis. Journal of receptor and signal transduction research:1-6

114. Kutay H et al. Downregulation of miR-122 in the rodent and human hepatocellular carcinomas. J Cell Biochem. 2006;99(3): 671-8.

115. Peters $\mathrm{U}$ et al. Vitamin $\mathrm{E}$ and selenium supplementation and risk of prostate cancer in the Vitamins and lifestyle (VITAL) study cohort. Cancer Causes Control : CCC. 2008;19(1):75-87.

116. Nian H, Bisson WH, Dashwood WM, Pinto JT, Dashwood RH. Alpha-keto acid metabolites of organoselenium compounds inhibit histone deacetylase activity in human colon cancer cells. Carcinogenesis. 2009;30(8):1416-23.

117. Lee JI et al. Alpha-keto acid metabolites of naturally occurring organoselenium compounds as inhibitors of histone deacetylase in human prostate cancer cells. Cancer Prev Res. 2009;2(7): 683-93.

118. Dashwood RH. Early detection and prevention of colorectal cancer (review). Oncol Rep. 1999;6(2):277-81.

119. Maciel-Dominguez A, Swan D, Ford D, Hesketh J. Selenium alters miRNA profile in an intestinal cell line: evidence that miR185 regulates expression of GPX2 and SEPSH2. Mol Nutr Food Res. 2013;57(12):2195-205.

120. Davidson LA et al. n-3 Polyunsaturated fatty acids modulate carcinogen-directed non-coding microRNA signatures in rat colon. Carcinogenesis. 2009;30(12):2077-84.

121. Farago N, Feher LZ, Kitajka K, Das UN, Puskas LG. MicroRNA profile of polyunsaturated fatty acid treated glioma cells reveal apoptosis-specific expression changes. Lipids Health Dis. 2011;10:173.

122.• Sethi S, Li Y, Sarkar FH. Regulating miRNA by natural agents as a new strategy for cancer treatment. Curr Drug Targets. 2013;14(10):1167-74. This review article offered the latest development on regulation of microRNAs by various natural chemotherapeutic agents. They have also provided evidence that these natural agents could inhibit cancer progression, and metastasis. In addition, these compounds will enhance drug sensitivity and reverse epithelial mesenchymal transition. This article supports the newer therapeutic approach for cancer treatment.

123. Subramaniam D et al. RNA binding protein CUGBP2/CELF2 mediates curcumin-induced mitotic catastrophe of pancreatic cancer cells. PLoS One. 2011;6(2):e16958.

124. Sun $\mathrm{M}$ et al. Curcumin (diferuloylmethane) alters the expression profiles of microRNAs in human pancreatic cancer cells. Mol Cancer Ther. 2008;7(3):464-73.

125. Bao B et al. Anti-tumor activity of a novel compound-CDF is mediated by regulating miR-21, miR-200, and PTEN in pancreatic cancer. PLoS One. 2011;6(3):e17850.

126. Saud SM, et al. (2014) Resveratrol prevents tumorigenesis in mouse model of Kras activated sporadic colorectal cancer by suppressing oncogenic Kras expression. Carcinogenesis.

127. Tili E, Michaille JJ. Resveratrol, MicroRNAs, Inflammation, and Cancer. J Nucleic Acids. 2011;2011:102431.

128. Tili E et al. Resveratrol decreases the levels of miR-155 by upregulating miR-663, a microRNA targeting JunB and JunD. Carcinogenesis. 2010;31(9):1561-6.

129. Tili E et al. Resveratrol modulates the levels of microRNAs targeting genes encoding tumor-suppressors and effectors of TGFbeta signaling pathway in SW480 cells. Biochem Pharmacol. 2010;80(12):2057-65.

130. Del Follo-Martinez A, Banerjee N, Li X, Safe S, Mertens-Talcott $\mathrm{S}$. Resveratrol and quercetin in combination have anticancer activity in colon cancer cells and repress oncogenic microRNA-27a. Nutr Cancer. 2013;65(3):494-504.

131. Kumazaki M et al. Anti-cancer effects of naturally occurring compounds through modulation of signal transduction and miRNA expression in human colon cancer cells. J Nutr Biochem. 2013;24(11):1849-58.

132. Bigelow RL, Cardelli JA. The green tea catechins, (-)Epigallocatechin-3-gallate (EGCG) and (-)-Epicatechin-3-gallate (ECG), inhibit HGF/Met signaling in immortalized and tumorigenic breast epithelial cells. Oncogene. 2006;25(13):1922-30.

133. Saldanha SN, Kala R, Tollefsbol TO. Molecular mechanisms for inhibition of colon cancer cells by combined epigeneticmodulating epigallocatechin gallate and sodium butyrate. Exp Cell Res. 2014;324(1):40-53.

134. Baselga-Escudero $\mathrm{L}$ et al. Resveratrol and EGCG bind directly and distinctively to miR-33a and miR-122 and modulate divergently their levels in hepatic cells. Nucleic Acids Res. 2014;42(2):882-92. 
135. Chakrabarti M, Khandkar M, Banik NL, Ray SK. Alterations in expression of specific microRNAs by combination of 4-HPR and EGCG inhibited growth of human malignant neuroblastoma cells. Brain Res. 2012;1454:1-13.

136. Tsang WP, Kwok TT. Epigallocatechin gallate up-regulation of miR-16 and induction of apoptosis in human cancer cells. J Nutr Biochem. 2010;21(2):140-6.

137. Luo Y, et al. (2014) Apoptotic effect of genistein on human colon cancer cells via inhibiting the nuclear factor-kappa B (NF-kappaB) pathway. Tumour biology: the journal of the International Society for Oncodevelopmental Biology and Medicine.

138. Chiyomaru T et al. Genistein up-regulates tumor suppressor microRNA-574-3p in prostate cancer. PLoS One. 2013;8(3): e58929.

139. Xia J et al. Genistein Inhibits Cell Growth and Invasion Through Regulation of miR-27a in Pancreatic Cancer Cells. Curr Pharm Des. 2014;20(33):5348-53.

140. Zaman MS et al. Inhibition of PTEN gene expression by oncogenic miR-23b-3p in renal cancer. PLoS One. 2012;7(11):e50203.

141. Gupta P, Kim B, Kim SH, Srivastava SK. Molecular targets of isothiocyanates in cancer: recent advances. Mol Nutr Food Res. 2014;58(8):1685-707.

142. Shan Y et al. Epithelial-mesenchymal transition, a novel target of sulforaphane via COX-2/MMP2, 9/Snail, ZEB1 and miR-200c/ ZEB1 pathways in human bladder cancer cells. J Nutr Biochem. 2013;24(6):1062-9.
143. Izzotti A et al. Modulation of microRNA expression by budesonide, phenethyl isothiocyanate and cigarette smoke in mouse liver and lung. Carcinogenesis. 2010;31(5):894-901.

144. Maruthanila VL, Poornima J, Mirunalini S. Attenuation of Carcinogenesis and the Mechanism Underlying by the Influence of Indole-3-carbinol and Its Metabolite 3,3'Diindolylmethane: A Therapeutic Marvel. Adv Pharmacol Sci. 2014;2014:832161.

145. Li $\mathrm{Y}$ et al. miR-146a suppresses invasion of pancreatic cancer cells. Cancer Res. 2010;70(4):1486-95.

146. Melkamu T, Zhang X, Tan J, Zeng Y, Kassie F. Alteration of microRNA expression in vinyl carbamate-induced mouse lung tumors and modulation by the chemopreventive agent indole-3carbinol. Carcinogenesis. 2010;31(2):252-8.

147. Paik WH et al. Chemosensitivity induced by down-regulation of microRNA-21 in gemcitabine-resistant pancreatic cancer cells by indole-3-carbinol. Anticancer Res. 2013;33(4):1473-81.

148. Sarkar $\mathrm{S}$ et al. Down-regulation of miR-221 inhibits proliferation of pancreatic cancer cells through up-regulation of PTEN, p27(kip1), p57(kip2), and PUMA. Am J Cancer Res. 2013;3(5): 465-77.

149. Le V, et al. (2014) Cytotoxic Effects of Ellagitannins Isolated from Walnuts in Human Cancer Cells. Nutrition and cancer:1-11

150. Wen XY et al. Ellagitannin (BJA3121), an anti-proliferative natural polyphenol compound, can regulate the expression of MiRNAs in HepG2 cancer cells. Phytother Res : PTR. 2009;23(6):778-84. 